MODELING, IDENTIFICATION AND CONTROL, 1994, VOL. 15, NO. 1, 23-32

doi:10.4173/mic.1994.13

\title{
A direct adaptive generalized predictive controller and some of its global convergence properties
}

\author{
WEI WANG† and ROLF HENRIKSEN $\ddagger$ \\ Keywords: Predictive control, adaptive control, convergence. \\ This paper is concerned with the direct approach of adaptive generalized predictive \\ control. Optimality of generalized predictive control is analyzed and an implicit \\ model with control law parameters is developed. A direct adaptive generalized \\ predictive control algorithm is suggested and some sufficient conditions for its \\ global convergence are also derived.
}

\section{Introduction}

During the last few years long-range predictive control (LRPC) has attracted increasing interest of several researchers. Many methods have been presented, see e.g. Ydstie (1984), Clarke et al. (1987), Lelic and Zarrop (1987), and De Keyser et al. (1988), which depend on the assumed model structure and the choice of cost function, including adaptive control methods, see Kramer and Unbehauen (1988) for an overview. Generalized predictive control (GPC) (Clarke et al. 1987; Clarke and Mohtadi 1989) is one of a new family of LRPC which has been used successfully in industrial applications, see Clarke (1988).

All LRPC approaches use a receding horizon strategy, i.e., a control sequence is computed by minimizing a cost function; then only the first element of the control sequence is applied to the plant and the whole procedure is repeated at the next sampling instant. It should be noted, however, that the receding horizon strategy does not ensure minimization of the original cost function. It is, therefore, natural to ask whether there is some cost function whose minimization with respect to the control variable will lead to the receding horizon strategy.

Another problem is that almost all the adaptive GPC algorithms appearing in the literature are in the indirect or explicit form. One disadvantage of the indirect form is that the computational load may be too heavy for on-line applications, especially in adaptive GPC algorithms because multi-step predictions are involved. To the best of the authors' knowledge, the only adaptive GPC controller in direct form for a deterministic plant reported in the literature is one where $N_{1}\left(N_{1}\right.$ is the prediction

Received 27 April 1993.

$\dagger$ Department of Automatic Control, Northeast University of Technology, Shenyang 110006, P.R. China. Currently on leave at The Norwegian Institute of Technology, Division of Engineering Cybernetics, N7034 Trondheim, Norway. The work was supported by the Royal Norwegian Council for Scientific and Industrial Research (NTNF) and the National Natural Science Foundation of China.

$\ddagger$ Norwegian Institute of Technology, Division of Engineering Cybernetics, N-7034 Trondheim, Norway.

This paper was presented at the 4th IFAC Symposium on Adaptive Systems in Control and Signal Processing, July 1-3, 1992, Grenoble. Reprinted with kind permission from Pergamon Press. 
horizon) parameter estimators are used at each sampling instant (Ortega and Sanchez, 1989).

In this paper the optimality of the GPC scheme is first established, and it is shown that the receding horizon control law can be obtained as a natural outcome of minimizing a cost function of generalized minimum variance type. Second, the work of Ortega and Sanchez (1989) is extended to the stochastic case, and we propose a direct adaptive GPC controller which utilizes only one parameter estimator. Finally, global convergence of the direct adaptive GPC algorithm is analysed under some assumptions.

\section{Generalized predictive control (GPC) scheme}

Consider a time-invariant plant described by the following CARIMA model

$$
A\left(z^{-1}\right) y(t)=B\left(z^{-1}\right) u(t-1)+C\left(z^{-1}\right) \omega(t) / \Delta
$$

where $A\left(z^{-1}\right), B\left(z^{-1}\right)$ and $C\left(z^{-1}\right)$ are polynomials in the backward shift operator $z^{-1}$ of the form

$$
\begin{aligned}
& A\left(z^{-1}\right)=1+a_{1} z^{-1}+\ldots+a_{u_{a}} z^{-n_{a}} \\
& B\left(z^{-1}\right)=b_{0}+b_{1} z^{-1}+\ldots+b_{u_{b}} z^{-n_{b}} \\
& C\left(z^{-1}\right)=1+c_{1} z^{-1}+\ldots+c_{n_{c}} z^{-n_{c}}
\end{aligned}
$$

$\{u(t)\},\{y(t)\}$ and $\{\omega(t)\}$ denote the plant input, output and disturbance sequences respectively. $\Delta=1-z^{-1}$ is the difference operator. Notice that the model (1) has the advantage that the controller will naturally contain an integrator. The sequence $\{\omega(t)\}$ is a stochastic process defined on a probability space $(\Omega, \mathscr{F}, \mathscr{P})$ on which we have a sequence of increasing sigma algebras $\left(\mathscr{F}_{t}, t \in \mathscr{N}\right)$ where $\mathscr{F}_{t}$ is generated by the observations up to and including time $t$. It is assumed that

$$
\begin{aligned}
\mathscr{E}\left\{\omega(t) \mid \mathscr{F}_{t-1}\right\} & =0 \quad \text { a.s. } \\
\mathscr{E}\left\{\omega(t)^{2} \mid \mathscr{F}_{t-1}\right\} & =\sigma^{2} \quad \text { a.s. } \\
\lim _{N \rightarrow \infty} \operatorname{Sup} \frac{1}{N} \sum_{t=1}^{N} \omega(t)^{2} & <\infty \quad \text { a.s. }
\end{aligned}
$$

The following assumptions will be made about the plant (1).

A1. The polynomial degrees $n_{a}, n_{b}$ and $n_{c}$ are known.

A2. $C\left(z^{-1}\right)$ is a stable polynomial.

The cost function has the following form

$$
J=\mathscr{E}\left\{\sum_{j=1}^{N_{1}}\left(y(t+j)-y_{r}(t+j)\right)^{2}+\lambda \sum_{j=1}^{N_{u}}(\Delta u(t+j-1))^{2} \mid \mathscr{F}_{t}\right\}
$$

where $\Delta u(t+j)=0, j=N_{u} \ldots, N_{1}$, and where $\left\{y_{r}(t)\right\}$ is a known bounded reference sequence. $N_{1}$ is the prediction horizon whereas $N_{u}$ is the control horizon. $\lambda$ is a weighting constant. The expectation in (5) is made given data obtained up to and including time $t$.

Using the following polynomial equations

$$
C\left(z^{-1}\right)=E_{j}\left(z^{-1}\right) A\left(z^{-1}\right) \Delta+z^{-j} F_{j}\left(z^{-1}\right)
$$




$$
E_{j}\left(z^{-1}\right) B\left(z^{-1}\right)=G_{j}\left(z^{-1}\right) C\left(z^{-1}\right)+z^{-j} H_{j}\left(z^{-1}\right)
$$

where $j=1,2, \ldots, N_{1}$ and

$$
\begin{aligned}
& E_{j}\left(z^{-1}\right)=e_{0}+e_{1} z^{-1}+\ldots+e_{j-1} z^{-j+1} \\
& F_{j}\left(z^{-1}\right)=f_{0}^{i}+f_{1}^{i} z^{-1}+\ldots+f_{n}^{i} z^{-n} \\
& G_{j}\left(z^{-1}\right)=g_{0}+g_{1} z^{-1}+\ldots+g_{j-1} z^{-j+1} \\
& H_{j}\left(z^{-1}\right)=h_{0}^{j}+h_{1}^{j} z^{-1}+\ldots+h_{m}^{j} z^{-m}
\end{aligned}
$$

where $n=\max \left(n_{a}, n_{c}-j\right)$ and $m=\max \left(n_{b}-1, n_{\mathrm{c}}-1\right)$, the plant (2.1) can be written in the form

$$
\begin{aligned}
y(t+j)= & G_{j} \Delta u(i+j-1)+\frac{F_{j}}{C} y(t) \\
& +\frac{H_{j}}{C} \Delta u(t-1)+E_{j} \omega(t+j)
\end{aligned}
$$

where $j=1, \ldots, N_{1}$.

The future output value $y(t+j)$ consists of future input signals, current input and output, and future noise signals. The term $E_{j} \omega(t+j)$ is uncorrelated with all other terms on the right-hand side of (10) if it is assumed that $u(t), \ldots, u\left(t+N_{1}-1\right)$ are $\mathscr{F}_{t^{-}}$ measurable.

Equation (10) can be written in vector form as

$$
\mathbf{y}=\mathbf{G u}+\frac{\mathbf{F}}{C} y(t)+\frac{\mathbf{H}}{C} \Delta u(t-1)+\mathbf{E}
$$

where

$$
\begin{aligned}
\mathbf{y}^{\mathrm{T}} & =\left[y(t+1) \ldots y\left(t+N_{1}\right)\right] \\
\mathbf{u}^{\mathrm{T}} & =\left[\Delta u(t) \ldots \Delta u\left(t+N_{u}-1\right)\right] \\
\mathbf{F}^{\mathrm{T}} & =\left[F_{1} \ldots F_{N_{1}}\right] \\
\mathbf{H}^{\mathrm{T}} & =\left[H_{1} \ldots H_{N^{1}}\right] \\
\mathbf{E}^{\mathrm{T}} & =\left[E_{1} \omega(t+1) \ldots E_{N_{1}} \omega\left(t+N_{1}\right)\right]
\end{aligned}
$$

and where $\mathbf{G}$ is the $N_{1} \times N_{u}$ lower-triangular matrix

$$
\mathbf{G}=\left[\begin{array}{cccc}
g_{0} & & & \\
g_{1} & g_{0} & & \\
\ldots & & & \\
g_{N_{u}-1} & g_{N_{u}-2} & \ldots & g_{0} \\
\ldots & & & \\
g_{N_{1}-1} & g_{N_{1}-2} & \ldots & g_{N_{1}-N_{u}}
\end{array}\right]
$$

Define

$$
\mathbf{y}_{r}^{\mathrm{T}}=\left[y_{r}(t+1) \ldots y_{r}\left(t+N_{1}\right)\right]
$$


From the definition of $\mathbf{y}, \mathbf{y}_{\mathbf{r}}$ and $\mathbf{u}$ the cost function (5) can be written as

$$
J=\mathscr{E}\left\{\left(\mathbf{y}-\mathbf{y}_{\mathbf{r}}\right)^{\mathbf{T}}\left(\mathbf{y}-\mathbf{y}_{\mathbf{r}}\right)+\lambda \mathbf{u}^{\mathbf{T}} \mathbf{u} \mid \mathscr{F}_{t}\right\}
$$

Substituting (11) into (13) and minimizing $J$ with respect to $u$ results in the control law

$$
\mathbf{u}=\left(\mathbf{G}^{\mathrm{T}} \mathbf{G}+\lambda \mathbf{I}\right)^{-1} \mathbf{G}^{\mathrm{T}}\left[\mathbf{y}_{r}-\frac{\mathbf{F}}{C} y(t)-\frac{\mathbf{H}}{C} \Delta u(t-1)\right]
$$

Writing the first row of $\left(\mathbf{G}^{\mathbf{T}} \mathbf{G}+\lambda \mathbf{I}\right)^{-1} \mathbf{G}^{\mathbf{T}}$ as

$$
\mathbf{P}^{\mathrm{T}}=\left[p_{1} \ldots p_{N 1}\right]
$$

we define

$$
P\left(z^{-1}\right)=p_{N_{1}}+p_{N_{1}-1} z^{-1}+\ldots+p_{1} z^{-N_{1}+1}
$$

Then, from (14) and the receding horizon strategy (Clarke et al. 1987) the control difference $\Delta u(t)$ is given by

$$
\begin{aligned}
\Delta u(t) & =\mathbf{P}^{\mathrm{T}}\left[\mathbf{y}_{r}-\frac{\mathbf{F}}{C} y(t)-\frac{\mathbf{H}}{C} \Delta u(t-1)\right] \\
& =P\left(z^{-1}\right) y_{r}\left(t+N_{1}\right)-l(t)
\end{aligned}
$$

where $l(t)$ satisfies

$$
C\left(z^{-1}\right) l(t)=\alpha\left(z^{-1}\right) y(t)+\beta\left(z^{-1}\right) \Delta u(t-1)
$$

Furthermore

$$
\begin{aligned}
& \alpha\left(z^{-1}\right)=\sum_{j=1}^{N_{1}} p_{j} F_{j}\left(z^{-1}\right) \\
& \beta\left(z^{-1}\right)=\sum_{j=1}^{N_{1}} p_{j} H_{j}\left(z^{-1}\right)
\end{aligned}
$$

where the degrees of $\alpha\left(z^{-1}\right)$ and $\beta\left(z^{-1}\right)$ are $n$ and $m$ respectively.

Substituting (17) into (1) gives the closed-loop system

$$
T y(t)=z^{-1} B C P y_{r}\left(t+N_{1}\right)+\left(C+z^{-1} \beta\right) C \omega(t)
$$

where

$$
T\left(z^{-1}\right)=A\left(z^{-1}\right) \Delta\left(C\left(z^{-1}\right)+z^{-1} \beta\left(z^{-1}\right)\right)+z^{-1} B\left(z^{-1}\right) \alpha\left(z^{-1}\right)
$$

Using (6) and (7), the closed-loop characteristic polynomial $T\left(z^{-1}\right)$ can be written as

$$
T\left(z^{-1}\right)=C\left(z^{-1}\right)\left(A\left(z^{-1}\right) \Delta+z^{-1} \sum_{j=1}^{N_{1}} z^{j} p_{j}\left(B\left(z^{-1}\right)-G_{j}\left(z^{-1}\right) A\left(z^{-1}\right) \Delta\right)\right)
$$

Hence, the closed-loop system has poles corresponding to the roots of $C\left(z^{-1}\right)$ and of

$$
T^{\prime}\left(z^{-1}\right)=A\left(z^{-1}\right) \Delta+z^{-1} \sum_{j=1}^{N_{1}} z^{j} p_{j}\left(B\left(z^{-1}\right)-G_{j}\left(z^{-1}\right) A\left(z^{-1}\right) \Delta\right)
$$

It should be noted from the above derivation that the control law (17) does not ensure minimization of $J$ since a receding horizon strategy has been used. 


\section{Optimality of GPC scheme}

It should be pointed out that the relationship between GPC and Linear Quadratic Gaussian (LQG) control has been established (Bitmead, Gevers and Wertz 1990) by using a state-space model and a Riccati difference equation, where the GPC control law is subsumed within the LQG framework as a receding horizon LQ tracking controller with specific choices for the cost function weighting matrices. In this section we will derive the receding horizon control law (17) in a different way. It will be shown that the receding horizon control law (17) can be obtained as a natural outcome of minimizing another cost function $J_{1}$, where $J_{1}$ has a similar structure as the general cost function of generalized minimum variance (GMV) control.

Let us denote the first row of $\left(\mathbf{G}^{\mathrm{T}} \mathbf{G}+\lambda \mathbf{I}\right)^{-1}$ by

$$
\mathbf{Q}^{\mathrm{T}}=\left[q_{1} \ldots q_{N_{u}}\right]
$$

and define

$$
Q\left(z^{-1}\right)=q_{N_{u}}+q_{N_{u}-1^{-1}} z^{-1}+\ldots+q_{1} z^{-N_{u}+1}
$$

Theorem 1. The receding horizon control law (17) minimizes the cost function

$$
J_{1}=\mathscr{E}\left\{\left(P\left(z^{-1}\right)\left(y\left(t+N_{1}\right)-y_{r}\left(t+N_{1}\right)\right)+\lambda Q\left(z^{-1}\right) \Delta u\left(t+N_{u}-1\right)\right)^{2} \mid \mathscr{F}_{t}\right\}
$$

where the polynomials $P\left(z^{-1}\right)$ and $Q\left(z^{-1}\right)$ are given by (16) and (22) respectively. Moreover, the minimum possible value of the quadratic cost function (23) is

$$
\gamma^{2}=\sigma^{2} \sum_{j=1}^{N_{1}}\left(\sum_{i=0}^{N_{1}-j} p_{i+j} e_{i}\right)^{2}
$$

Proof: For the first part, multiplying by $\mathbf{G}^{\mathrm{T}}$ and adding $\lambda \mathbf{I u}$ on both sides of equation (11) we obtain

$$
\mathbf{G}^{\mathrm{T}} \mathbf{y}+\lambda \mathbf{I} \mathbf{u}=\mathbf{G}^{\mathrm{T}} \mathbf{G u}+\lambda \mathbf{I} \mathbf{u}+\mathbf{G}^{\mathrm{T}}\left(\frac{\mathbf{F}}{C} y(t)+\frac{\mathbf{H}}{C} \Delta u(t-1)+\mathbf{E}\right)
$$

which results in

$$
\mathbf{u}=\left(\mathbf{G}^{\mathbf{T}} \mathbf{G}+\lambda \mathbf{I}\right)^{-1}\left[\mathbf{G}^{\mathrm{T}}\left(\mathbf{y}-\mathbf{E}-\frac{\mathbf{F}}{C} y(t)-\frac{\mathbf{H}}{C} \Delta u(t-1)\right)+\lambda \mathbf{I} \mathbf{u}\right]
$$

Using (16), (18), (19) and (22) the first row of vector equation (26) can be written as

$$
\Delta u(t)=P\left(z^{-1}\right) y\left(t+N_{1}\right)+\lambda Q\left(z^{-1}\right) \Delta u\left(t+N_{u}-1\right)-v\left(t+N_{1}\right)-l(t)
$$

where

$$
v\left(t+N_{1}\right)=\sum_{j=1}^{N_{1}} p_{j} E_{j}\left(z^{-1}\right) \omega(t+j)
$$

and where $E_{j}\left(z^{-1}\right)$ is given in (8). Define

$$
\phi\left(t+N_{1}\right)=P\left(z^{-1}\right) y\left(t+N_{1}\right)+\lambda Q\left(z^{-1}\right) \Delta u\left(t+N_{u}-1\right)
$$

Then (27) can be written as

$$
\phi\left(t+N_{1}\right)-v\left(t+N_{1}\right)=l(t)+\Delta u(t)
$$


We note that $\phi\left(t+N_{1}\right)-v\left(t+N_{1}\right)$ is $\mathscr{F}_{t}$-measurable. It is obvious that $\phi\left(t+N_{1}\right)-$ $v\left(t+N_{1}\right)$ is the optimal linear prediction of $\phi\left(t+N_{1}\right)$ given $\mathscr{F}_{t}$, i.e.

$$
\phi^{0}\left(t+N_{1}\right)=\phi\left(t+N_{1}\right)-v\left(t+N_{1}\right)=l(t)+\Delta u(t)
$$

Now, note that

$$
\phi\left(t+N_{1}\right)=\phi^{0}\left(t+N_{1}\right)+v\left(t+N_{1}\right)
$$

Substituting (29), (31) and (32) into (23) we obtain after some manipulations

$$
J_{1}=\mathscr{E}\left\{\left(l(t)+\Delta u(t)-P y_{r}\left(t+N_{1}\right)\right)^{2}\right\}+\mathscr{E}\left\{v\left(t+N_{1}\right)^{2} \mid \mathscr{F}_{t}\right\} \geqslant \mathscr{E}\left\{v\left(t+N_{1}\right)^{2} \mid \mathscr{F}_{i}\right\}
$$

The first term on the right-hand side of (33) is greater or equal to zero and is brought to zero by the control action given in (17). This completes the first part.

From (28) we finally obtain

$$
\begin{aligned}
\mathscr{E}\left\{v\left(t+N_{1}\right)^{2} \mid \mathscr{F}_{t}\right\} & =\mathscr{E}\left\{\left(\sum_{j=1}^{N_{1}} p_{j} \sum_{i=0}^{j-1} e_{i} \omega(t+j-i)\right)^{2} \mid \mathscr{F}_{t}\right\} \\
& =\sigma^{2} \sum_{j=1}^{N_{1}}\left(\sum_{i=0}^{N_{1}-j} p_{i+j} e_{i}\right)^{2}=\gamma^{2}
\end{aligned}
$$

$\nabla \nabla \nabla$

Comparing the cost function $J_{1}$ given above with the cost function used in GMV control (Clarke and Gawthrop 1975, 1979), we see that there are future control signals in $J_{1}$. This is the reason for adding $\lambda Q \Delta u\left(t+N_{u}-1\right)$ to $\phi(t)$ in (29). If we take $N_{u}=1$, then $Q\left(z^{-1}\right)=q_{1}$ and

$$
J_{1}=\mathscr{E}\left\{\left(P\left(z^{-1}\right)\left(y\left(t+N_{1}\right)-y_{r}\left(t+N_{1}\right)\right)+\lambda^{\prime} \Delta u(t)\right)^{2} \mid \mathscr{F}_{t}\right\}
$$

where $\lambda^{\prime}=\lambda q_{1}$. In this case $J_{1}$ is just a special form of the cost function in GMV control. It is clear that in GPC the polynomials $P$ and $Q$ cannot be chosen arbitrarily; they will depend on the plant parameters $a_{i}$ and $b_{i}$, the prediction and control horizons $N_{1}$ and $N_{u}$, and the weighting constant $\lambda$. For a given plant the stability of the closed-loop system will thercfore depend on the choices of $N_{1}, N_{u}$ and $\lambda$.

In the following section we will see that an implicit model for direct GPC control can be derived by the use of $\phi(t)$ in (29).

\section{Direct adaptive GPC controller}

The direct adaptive GPC controller and the global convergence analysis given in the sequel are based on the following assumptions in addition to assumptions A1 and A2.

A3. The closed-loop system's characteristic polynomial

$$
T^{\prime}\left(z^{-1}\right)=A\left(z^{-1}\right) \Delta+z^{-1} \sum_{j=1}^{N_{1}} z^{j} p_{j}\left(B\left(z^{-1}\right)-G_{j}\left(z^{-1}\right) A\left(z^{-1}\right) \Delta\right)
$$

is stable, i.e., $T^{\prime}(q) \neq 0$ for $|q|<1$.

A4. The first $N_{1}$ terms of the plant's step response, i.e. $g_{0}, g_{1}, \ldots, g_{N_{1}-1}$, are known.

A5. $C\left(z^{-1}\right)-\bar{\alpha} / 2$ is strictly positive real for some positive $\bar{\alpha}$. 
Remark 1: The assumption A3 means that when the plant's parameters are known, the receding horizon control law (17) with suitable choices of $N_{1}, N_{u}$ and $\lambda$ stabilizes the plant.

Remark 2: The assumption A4 used here is similar to the one used by Ortega and Sanchez (1989), where knowledge of the first $N_{1}$ terms of the plant's impulse response are required because a CARIMA model is used. In our adaptive GPC algorithm and the convergence analysis following hereafter we take (30) to be an equation which is estimated on-line, which implies that $\phi(t)$ will be available at time $t$. We know from equations (6) and (7) that the coefficients of $G_{j}\left(z^{-1}\right)$, i.e., $g_{0}, g_{1}, \ldots, g_{N_{1}-1}$ are simply the first $N_{1}$ terms of the plant's step response. If $g_{0}, g_{1}, \ldots, g_{N_{1}-1}$ are known and $\lambda$ is given, then the coefficients of $P\left(z^{-1}\right)$ and $Q\left(z^{-1}\right)$ can be computed off-line.

Note that (30) can be written as

$$
\begin{aligned}
C\left(z^{-1}\right)\left(\phi\left(t+N_{1}\right)-\right. & \left.v\left(t+N_{1}\right)\right) \\
& =\alpha\left(z^{-1}\right) y(t)+\beta\left(z^{-1}\right) \Delta u(t-1)+C\left(z^{-1}\right) \Delta u(t) \\
& =\alpha\left(z^{-1}\right) y(t)+\beta^{\prime}\left(z^{-1}\right) \Delta u(t-1)+\Delta u(t)
\end{aligned}
$$

where

$$
\beta^{\prime}\left(z^{-1}\right)=\beta\left(z^{-1}\right)+z\left(C\left(z^{-1}\right)-1\right)
$$

Subtracting $C\left(z^{-1}\right) P\left(z^{-1}\right) y_{r}\left(t+N_{1}\right)$ from both sides of (36) we have

where

$$
\begin{aligned}
C\left(\phi\left(t+N_{1}\right)-P y_{r}(\right. & \left.\left.+N_{1}\right)-v\left(t+N_{1}\right)\right) \\
& =\alpha y(t)+\beta^{\prime} \Delta u(t-1)-C P y_{r}\left(t+N_{1}\right)+\Delta u(t) \\
& =X(t)^{\mathrm{T}} \theta+\Delta u(t)-P y_{r}\left(t+N_{1}\right)
\end{aligned}
$$

$$
\begin{aligned}
X(t)^{\mathrm{T}}= & {[y(t), \ldots, y(t-n), \Delta u(t-1), \ldots, \Delta u(t-m-1)} \\
& \left.-P y_{r}\left(t+N_{1}-1\right), \ldots,-P y_{r}\left(t+N_{1}-n_{c}\right)\right] \\
\theta^{\mathrm{T}}= & {\left[\alpha_{0}, \alpha_{1}, \ldots, \alpha_{m}, \beta_{0}^{\prime}, \ldots, \beta_{m}^{\prime}, c_{1}, \ldots, c_{n_{c}}\right] }
\end{aligned}
$$

When the parameter vector $\theta$ is unknown we have to use an estimate $\theta(t)$ instead of $\theta$ at time $t$. For estimation of $\theta$ we use the estimation algorithm given by Goodwin $e t$ al. (1980), viz.

$$
\begin{gathered}
\varepsilon(t)=\phi(t)-\Delta u\left(t-N_{1}\right)-X\left(t-N_{1}\right)^{\mathrm{T}} \hat{\theta}\left(t-N_{1}\right) \\
\hat{\theta}(t)=\hat{\theta}\left(t-N_{1}\right)+\frac{\bar{\alpha}}{r\left(t-N_{1}\right)} X\left(t-N_{1}\right) \varepsilon(t) \\
r\left(t-N_{1}\right)=r\left(t-N_{1}-1\right)+X\left(t-N_{1}\right)^{\mathrm{T}} X\left(t-N_{1}\right)
\end{gathered}
$$

where $\bar{\alpha}>0$ and $r(0)=1$.

All this yields the following direct adaptive GPC algorithm.

Data: Given the prediction horizon $N_{1}$, the control horizon $N_{u}$, the weighting constant $\lambda$ and the first $N_{1}$ terms of the plant's step response, i.e. $\mathrm{g}_{0}, g_{1}, \ldots, g_{N_{1}-1}$.

Step 1: Compute $\phi(t)$ from

$$
\phi(t)=P\left(z^{-1}\right) y(t)+\lambda Q\left(z^{-1}\right) \Delta u\left(t-N_{1}+N_{u}-1\right)
$$


Step 2: Compute $\hat{\theta}(t)$ from (38)-(40).

Step 3: Compute the control $u(t)$ from

$$
u(t)=u(t-1)+P\left(z^{-1}\right) y_{r}\left(t+N_{1}\right)-X(t)^{\mathrm{T}} \hat{\theta}(t)
$$

Step 4: Increase $t$ by one and return to the step 1 .

Note that the estimation scheme (a stochastic approximation) used in this algorithm is arbitrary; it is chosen simply to illustrate the proof technique in the following analysis. Other estimation schemes, e.g., projection or least squares schemes, can of course also be used in the above algorithm.

\section{Convergence analysis}

We start with an asymptotic property possessed by the estimation algorithm (38)(40). Define

$$
\begin{aligned}
\widetilde{\theta}(t) & =\hat{\theta}(t)-\theta \\
e(t) & =\phi(t)-P\left(z^{-1}\right) y_{r}(t) \\
z\left(t-N_{1}\right) & =e(t)-v(t) \\
V(t) & =\widetilde{\theta}(t)^{\mathrm{T}} \tilde{\theta}(t)
\end{aligned}
$$

From (37), (42), (44) and (45) we have

$$
C\left(z^{-1}\right) z\left(t-N_{1}\right)=-X\left(t-N_{1}\right)^{\mathrm{T}} \tilde{\theta}\left(t-N_{1}\right)
$$

Lemma 1: Subject to assumptions A1-A5, the estimation algorithm (38)-(40) has the following property:

$$
\lim _{N \rightarrow \infty} \frac{N}{r(N)} \cdot \frac{1}{N} \sum_{t=1}^{N} z(t)^{2}=0 \text { a.s. }
$$

Proof: See Goodwin et al. (1980).

Theorem 2. Subject to assumptions A1-A5, if the adaptive GPC algorithm given in the above section is applied to the plant (1), one has, a.s.,

S1) $\lim _{N \rightarrow \infty} \sup \frac{1}{N_{t}} \sum_{t=1}^{N} y(t)^{2}<\infty$

S2) $\lim _{N \rightarrow \infty} \sup \frac{1}{N_{t}} \sum_{t=1}^{N}(\Delta u(t))^{2}<\infty$

S3) $\lim _{N \rightarrow \infty} \frac{1}{N_{t}} \sum_{t=N_{1}}^{N} \mathscr{E}\left\{\left(P\left(z^{-1}\right)\left(y(t)-y_{r}(t)\right)\right)+\left(\lambda Q\left(z^{-1}\right) \Delta u\left(t-N_{1}+N_{u}-1\right)\right)^{2} \mid \mathscr{F}_{t-N_{1}}\right\}=\gamma^{2}$

Proof: From (36), (44) and (45) we have

$$
C\left(z^{-1}\right) z(t)=\alpha\left(z^{-1}\right) y(t)+\left(C\left(z^{-1}\right)+z^{-1} \beta\left(z^{-1}\right)\right) \Delta u(t)-C\left(z^{-1}\right) P\left(z^{-1}\right) y_{r}\left(t+N_{1}\right)
$$

Multiplying (49) by $\triangle A\left(z^{-1}\right)$ and using (1), (6) and (7) lead to

$$
T^{\prime}\left(z^{-1}\right) \Delta u(t)=\Delta A\left(z^{-1}\right) z(t)+\Delta A\left(z^{-1}\right) P\left(z^{-1}\right) y_{r}\left(t+N_{1}\right)-\alpha\left(z^{-1}\right) \omega(t)
$$


Multiplying (49) by $B\left(z^{-1}\right)$ and using (1), (6) and (7) result in

$$
T^{\prime}\left(z^{-1}\right) y(t+1)=B\left(z^{-1}\right) z(t)+B\left(z^{-1}\right) P\left(z^{-1}\right) y_{r}\left(t+N_{1}\right)+\left(C\left(z^{-1}\right)+z^{-1} B\left(z^{-1}\right)\right) \omega(t+1)
$$

Since $T^{\prime}\left(z^{-1}\right)$ is stable and $y_{r}(t)$ is bounded, it is clear from (4), (50) and (51) that there exists a $N^{\prime}$ such that, for $N>N^{\prime}$,

and

$$
\frac{1}{N} \sum_{t=1}^{N}(\Delta u(t))^{2} \leqslant \frac{K_{1}}{N} \sum_{t=1}^{N} z(t)^{2}+K_{2}
$$

$$
\frac{1}{\mathrm{~N}} \sum_{t=1}^{N} y(t+1)^{2} \leqslant \frac{K_{3}}{N} \sum_{t=1}^{N} z(t)^{2}+K_{4}
$$

From the definition of $X(t)$ and $r(t)$ there exists a $N^{\prime \prime}$ such that, for $N>N^{\prime \prime}$,

$$
\frac{r(N)}{N} \leqslant \frac{K_{5}}{N} \sum_{t=1}^{N} y(t+1)^{2}+\frac{K_{6}}{N} \sum_{t=1}^{N}(\Delta u(t))^{2}+K_{7}
$$

Using (52)-(54) we have

$$
\frac{r(N)}{N} \leqslant \frac{C_{1}}{N_{t}} \sum_{t=1}^{N} z(t)^{2}+C_{2}
$$

for $N>\bar{N}$ where $\bar{N}=\max \left(N^{\prime}, N^{\prime \prime}\right)$.

Now from (48) and (55) we conclude that

$$
\lim _{N \rightarrow \infty} \frac{\frac{1}{N_{t}} \sum_{t=1}^{N} z(t)^{2}}{\frac{C_{1}}{N_{t}} \sum_{t=1}^{N} z(t)^{2}+C_{2}}=0 \text { a.s. }
$$

and hence

$$
\lim _{N \rightarrow \infty} \frac{1}{N} \sum_{t=1}^{N} z(t)^{2}=0 \quad \text { a.s. }
$$

The results S1) and S2) now follow from (57), (52) and (53).

From (45) and (57) we obtain

$$
\lim _{N \rightarrow \infty} \frac{1}{N} \sum_{t=1}^{N}(e(t)-v(t))^{2}=0 \quad \text { a.s. }
$$

But from (28), (45) and (47) we know that $v(t)$ is a moving average of $\omega(t), \ldots$, $\omega\left(t-N_{1}+1\right)$, and $e(t)-v(t)$ is $\mathscr{F}_{t-N_{1}}$-measurable, whence

$$
\begin{aligned}
\mathscr{E}\left\{e(t)^{2} \mid \mathscr{F}_{t-N_{1}}\right\} & =\mathscr{E}\left\{(e(t)-v(t)+v(t))^{2} \mid \mathscr{F}_{t-N_{1}}\right\} \quad \text { a.s. } \\
& =(e(t)-v(t))^{2}+\mathscr{E}\left\{v(t)^{2} \mid \mathscr{F}_{t-N_{1}}\right\} \text { a.s. }
\end{aligned}
$$

From (34), (58) and (59)

$$
\lim _{N \rightarrow \infty} \frac{1}{N_{t}} \sum_{t=N_{1}}^{N} \mathscr{E}\left\{e(t)^{2} \mid \mathscr{F}_{t-N_{1}}\right\}=\mathscr{E}\left\{v(t)^{2} \mid \mathscr{F}_{t-N_{1}}\right\}=\gamma^{2} \quad \text { a.s. }
$$

The result S3) follows from (29), (44) and (60) which completes the proof. 


\section{Conclusion}

In this paper a direct adaptive GPC controller has been proposed, and global convergence of the algorithm has been proved by using standard analysis techniques under the main assumption that the first $N_{1}$ coefficients of the plant's step response are known. The motivation for this assumption is primarily that it allows us to analyse the convergence and stability properties of this type of adaptive controller. When the first $N_{1}$ coefficients of the plant's step response are imprecisely known, our direct adaptive GPC controller can be considered as an adaptive controller with unmodeled dynamics, and the convergence analysis can be carried out by using a dead zone and normalization techniques in the estimation scheme. This research is currently going on.

\section{REFERENCES}

BITMEAD, R. R., GEVERS, M. and WERTZ, V. (1990). Adaptive optimal control, the thinking man's GPC. (Prentice Hall, Englewood Cliffs, NJ).

Clarke, D. W. and Gawthrop, P. J. (1975). Self-tuning controller. Proc. IEE-D, 122, 929-934.

Clarke, D. W. and Gawthrop, P. J. (1979). Self-tuning control. Proc. IEE-D, 126, 633-640.

Clarke, D. W., Mohtadi, C. and TuFFs, P. S. (1987). Generalized predictive control-Part I and II. Automatica, 23, 137-160.

Clarke, D. W. (1988). Application of generalized predictive control. Proc. IF AC Adaptive Control of Chemical Processes, Copenhagen, pp. 1-8.

Clarke, D. W. and MohTAd, C.(1989). Properties of generalized predictive control. Automatica, 25, 859-875.

De Keyser, R. M. C., Van de Velde, Ph. G. A. and Dumortier, F. A. G. (1988). A comparative study of self-adaptive long-range predictive control methods. Automatica, 24, 149-163.

Goodwin, G. C., Sin, K. S., and SALUJA, K. K. (1980). Stochastic adaptive control and prediction: the general delay-colored noise case. IEEE Trans., AC-25, 946-950.

Kramer, K. and Unbehauen, H. (1988). Survey to adaptive long-range predictive control. Proc. 12th IM ACS World Congress on Scientific Computation, Paris, pp. 358-362.

LELIC, M. A. and ZARROP, M. B. (1987. Generalized pole-placement self-tuning controller-Part I and II. Int. J. Control, 46, 548-568.

ORTEGA, R. and SANCHEZ (1989). Globally convergent multistep receding horizon adaptive controller. Int. J. Control, 49, 1655-1664.

Yostie, B. E. (1984). Extended horizon adaptive control. Proc. IFAC 9th World Congress, Budapest, VII. pp. 133-137. 